\title{
Universal Linked Multiple Access Source Codes
}

\author{
Sidharth Jaggi Michelle Effros \\ Department of Electrical Engineering, California Institute of Technology \\ Mail Code 136-93, Pasadena, CA 91125, U.S.A. \\ email: $\{$ jaggi, effros\}@caltech.edu
}

\begin{abstract}
We consider the multiple access source coding (MASC) problem (also known as the SlepianWolf problem) for situations where the joint source statistics are unknown a priori. Since neither encoder receives information about the joint source statistics, we allow an asymptotically negligible amount of communication between the encoders. We prove the existence of universal 2-encoder Linked MASCs (LMASCs) with rates approaching the Slepian-Wolf bound, demonstrate the tightness of this bound, and calculate the rate of convergence of the proposed universal LMASC. The result generalizes to $M>2$ encoders. We also consider scenarios where the number of bits passed between the system encoders is allowed to grow linearly in the code dimension; in these scenarios one encoder can act as a conduit for the flow of another encoder's information.
\end{abstract}

\section{INTRODUCTION}

Given finite-alphabet sources $(X, Y) \in \mathcal{X} \times \mathcal{Y}$ drawn i.i.d. from p.m.f. $Q=\{q(x, y)\}$, a blocklength- $n, m$ round 2-encoder LMASC $\breve{C}_{n}=\left(l_{m, n}=\left(l_{m, n}^{(X)}, l_{m, n}^{(Y)}\right), f_{n}=\right.$ $\left.\left(f_{n}^{(X)}, f_{n}^{(Y)}\right), g_{n}\right)$ for $(X, Y)$ comprises two link encoders, two message encoders and a decoder. The link passes $m$ rounds of messages between encoders, and the link encoders at a source observe the source sequence and the previous link messages. That is, for each $k \in\{1, \ldots, m\}$, the link encoders are $l_{k, n}^{(X)}: \mathcal{X}^{n} \times \mathcal{J}^{k-1} \rightarrow \mathcal{I}_{k}$ and $l_{k, n}^{(Y)}: \mathcal{Y}^{n} \times \mathcal{I}^{k-1} \rightarrow \mathcal{J}_{k}$. The message encoders are Xavier $\left(f_{n}^{(X)}: \mathcal{X}^{n} \times \mathcal{J}^{m} \rightarrow S_{X}\right)$ and Yvonne $\left(f_{n}^{(Y)}: \mathcal{Y}^{n} \times \mathcal{I}^{m} \rightarrow S_{Y}\right)$, and the decoder is Zorba $\left(g_{n}: S_{X} \times S_{Y} \rightarrow \mathcal{X}^{n} \times \mathcal{Y}^{n}\right)$. Here $S_{X}, S_{Y}$, and $\mathcal{I}_{k}, \mathcal{J}_{k}$ $(k \in\{1, \cdots, m\})$ are binary prefix codes. The error probability, inter-encoder rate, and decoder input rate are defined as, respectively,

$$
\begin{aligned}
P_{e}\left(\breve{C}_{n}, Q\right) & =Q^{n}\left(g_{n}\left(f_{n}\left(X^{n}, Y^{n}\right)\right) \neq\left(X^{n}, Y^{n}\right)\right) \\
r\left(\breve{C}_{n}, Q\right) & =\frac{1}{n} E_{Q}\left(\Sigma_{k=1}^{m}\left|I_{k}\right|, \Sigma_{k=1}^{m}\left|J_{k}\right|\right) \\
R\left(\breve{C}_{n}, Q\right) & =\left(R_{X}\left(\breve{C}_{n}, Q\right), R_{Y}\left(\breve{C}_{n}, Q\right)\right) \\
& =\frac{1}{n} E_{Q}\left(\left|f_{n}^{(X)}\left(X^{n}, J^{m}\right)\right|,\left|f_{n}^{(Y)}\left(Y^{n}, I^{m}\right)\right|\right) .
\end{aligned}
$$

where $\left(I_{k}, J_{k}\right)=l_{k, n}\left(X^{n}, Y^{n}\right)$. Rate pair $\left(R_{X}, R_{Y}\right)$ is said to be $r_{X \leftrightarrow Y}$-LMASC-achievable on $Q$ if there exists a sequence $\left\{\breve{C}_{n}\right\}$ of LMASCs (possibly dependent on $Q$ ) such that $R\left(\breve{C}_{n}, Q\right) \rightarrow\left(R_{X}, R_{Y}\right), r\left(\breve{C}_{n}, Q\right) \rightarrow r_{X \leftrightarrow Y}=\left(r_{X \rightarrow Y}, r_{Y \rightarrow X}\right)$ and $P_{e}\left(\breve{C}_{n}, Q\right) \rightarrow 0$. The set of $r_{X \leftrightarrow Y}$-LMASC-achievable rates on $Q$ is denoted by $\mathcal{R}_{L}\left(Q, r_{X \leftrightarrow Y}\right)$. A sequence $\left\{\breve{C}_{n}\right\}_{n=1}^{\infty}$ of $r_{X \leftrightarrow Y}$-LMASCs is universal with respect to the class $\Omega$

${ }^{1}$ This material is based upon work partially supported by a Caltech E\&AS Division Fellowship, the NSF under Award No. CCR9909026 and by the Caltech Lee Center for Advanced Networking. of memoryless distributions on $\mathcal{X} \times \mathcal{Y}$ if, for every $Q \in$ $\Omega$, some point $\left(R_{X}^{\star}(Q), R_{Y}^{\star}(Q)\right)$ on the lower boundary of $\mathcal{R}_{L}\left(Q, r_{X \leftrightarrow Y}\right)$ is $r_{X \leftrightarrow Y}$-LMASC-achievable on $Q$ by $\left\{\breve{C}_{n}\right\}$.

\section{Main Result}

We here outline bounds on the performance of universal LMASCs. A brief sketch of the corresponding proofs follows. Details appear in [2].

Theorem 1 For any point $\left(R_{X}, R_{Y}\right)$ in $\mathcal{R}_{L}\left(Q, r_{X \leftrightarrow Y}\right)$ there exists a universal sequence $\left\{\breve{C}_{n}^{L}\right\}$ of $r_{X \leftrightarrow Y}-L M A S C s$ for source class $\Omega$. The $r_{X \leftrightarrow Y}-L M A S C$-achievable region is given by

$$
\begin{aligned}
R_{L}\left(Q, r_{X \leftrightarrow Y}\right)=\left\{\left(R_{X}, R_{Y}\right): R_{X}\right. & \geq H_{Q}(X \mid Y)-r_{X \rightarrow Y}, \\
R_{Y} & \geq H_{Q}(Y \mid X)-r_{Y \rightarrow X}, \\
R_{X}+R_{Y} & \left.\geq H_{Q}(X, Y)\right\} .
\end{aligned}
$$

Remarks: (1) Like the Slepian-Wolf Theorem [1], Theorem 1 can be generalized to $M$ encoders.

(2) The Slepian-Wolf region is the same as $\mathcal{R}_{L}(Q,(0,0))$.

The main argument constructs a universal sequence of 2-round $(0,0)$-LMASCs with $\left(R_{X}^{\star}(Q), R_{Y}^{\star}(Q)\right)=$ $\left(H_{Q}(X), H_{Q}(Y \mid X)\right)$. The symmetry of the problem makes $\left(H_{Q}(X \mid Y), H_{Q}(Y)\right)$ similarly attainable. Time-sharing between the codes achieving these two rate pairs or padding the codes with extraneous symbols gives every other rate pair in $\mathcal{R}_{L}(Q,(0,0))$. These $(0,0)$-LMASCs are then used to construct universal $r_{X \leftrightarrow Y}$-LMASCs.

Given the above scenario, we choose $l_{m, n}$ and $m(n)$ so that $\left(I^{m}, J^{m}\right)=\left(x^{m(n)}, y^{m(n)}\right)$ for some $m(n)$ satisfying $\lim _{n \rightarrow \infty}(m(n) / n)=0$. Then Xavier and Yvonne can independently make an estimate of $Q$ as $\hat{P}_{n}=\hat{p}_{n}(x, y)=$ $(1 / m(n)) \sum_{i=1}^{m(n)} 1\left(\left(X_{i}, Y_{i}\right)=(x, y)\right)$. We show in [2] that $Q^{m(n)}\left((x, y) \in \mathcal{X} \times \mathcal{Y}:\left|\hat{p}_{n}(x, y)-q(x, y)\right| \geq \delta(n)\right) \leq$ $2^{-m(n) \delta^{2}(n) c}$ (where $c$ is a constant independent of $Q$ ). Thus choosing $\delta(n)$ so that $\delta(n) \rightarrow 0$ and $m(n) \delta^{2}(n) \rightarrow \infty$ ensures that $\hat{P}_{n} \rightarrow Q$ with a probability that approaches 1 . We then use $\hat{P}_{n}$ to select a $\dot{P} \in \Omega$ and a corresponding code $\breve{C}_{n}(\dot{P})$ such that for $\Delta(n)=c^{\prime} \delta(n) \log (\delta(n))$ and any $Q \in \Omega\left(c^{\prime}\right.$ is a constant independent of $Q$ )

$$
\begin{aligned}
P_{e}\left(\breve{C}_{n}(\dot{P}), Q\right) & \leq 2^{-m(n) \delta^{2}(n) c+1} \\
r\left(\breve{C}_{n}(\dot{P}), Q\right) & =\frac{1}{n}(\lceil\log |\mathcal{X}| m(n)\rceil,\lceil\log |\mathcal{X}| m(n)\rceil) \\
R_{X}\left(\breve{C}_{n}(\dot{P}), Q\right) & \leq R_{X}^{\star}(Q)+\Delta(n) \\
R_{Y}\left(\breve{C}_{n}(\dot{P}), Q\right) & \leq R_{Y}^{\star}(Q)+\Delta(n)
\end{aligned}
$$

\section{REFERENCES}

[1] D. Slepian and J. K. Wolf, "Noiseless coding of correlated information sources," IEEE Transactions on Information Theory, vol. 19 , pp. 471-480, July 1973.

[2] S. Jaggi and M. Effros, "Universal linked multiple access source codes." In preparation for submission to the IEEE Transactions on Information Theory, 2002. 